\title{
Implementation of prediction of ball position in robot soccer using Kalman Filter
}

\author{
YUAN Cheng ${ }^{1, a^{*}, \text { LI Shuqin }}{ }^{2, b}$ \\ ${ }^{1}$ School of computer, Beijing Information Science\& Technology University, Beijing, P.R. China, \\ ${ }^{2}$ School of computer, Beijing Information Science\& Technology University, Beijing, P.R. China, \\ aych_327@hotmail.com, ${ }^{\text {b }}$ Lishuqin_de@126.com
}

Keywords: Kalman Filter, Robot Soccer, FIRA 5v5, state equation

Abstract. In the robot soccer competition whether able to accurately predict the ball's status direct impact on the completeness of the robot's motion, also determining the result of the game. In this paper, we have using the Kalman Filter to optimize the status data collected by the system, eliminate the noises in the data, implement a more accurate prediction of the ball status in several time periods.

\section{Introduction}

Since the establish of FIRA (Federation of International Robot-soccer Association) in 1997, many regions have also established their regional associations [1]. All kinds of robot soccer competitions have been held in everywhere around the world. The unique combination of the competition and academic research has stimulated strong interest in young students. Students have trained their rigorous scientific attitude and well skills.

The research of robot soccer system involves a very wide range of fields, including robotics, intelligent control, computer vision, computer graphics, artificial intelligence. The booming robot soccer competitions played an important role in promoting the research of robots.

Kalman filter, also known as linear quadratic estimation (LQE), is an algorithm that uses a series of measurements observed over time, containing statistical noise and other inaccuracies, and produces estimates of unknown variables that tend to be more precise than those based on a single measurement alone.

In the robot soccer competition, whether or not the goalkeeper could catch the ball or other robot could pass or shoot depends on the status of the ball, including position and velocity. So the prediction of the ball's position is the key of the game. By using Kalman filter we could get a more accurate status of the ball.

\section{Design philosophy}

In the robot soccer competition, even if the motion of soccer is diverse, as the time fragment is small, we can consider that the motion is linear in short time[2]. Meanwhile, the ball is at high speed, and it is a complicated process because of acceleration and deceleration, the location information obtained from the digital camera is extremely easy to lose. In addition, because of interfering, it cannot avoid having noise in the information thus it weakens the effect of control[3]. Regarding to the variance of data obtained from the system, using the Kalman filter to do the regression analysis on the motion of soccer, which can effectively eliminate the result caused by the noise when getting the information of motion, so it can increase the efficiency of achieving many strategies in robots soccer competitions.

In the robot soccer competition, the acquisition and update of data are very quick. So we ignored the impact of the acceleration of velocity and position. Introducing a matrix variable $\mathrm{X}$ to express ball status. At time T, the robot's position in the direction of X-axis is $S_{x}(t)$, the position in the direction of $\mathrm{Y}$ axis is $S_{y}(t)$. Meanwhile velocity is $V_{x}(\mathrm{t}), V_{y}(t)$. So 
$S(t)=S(t-1)+V(t-1) T$

$V(t)=V(t-1)$.

$\mathrm{T}$ is the update period of the position in the system, and

$$
\mathrm{X}(\mathrm{t})=\left[\begin{array}{ll}
S_{x}(t) & S_{y}(t) \\
V_{x}(t) & V_{y}(t)
\end{array}\right]
$$

Base on equations (1)(2)(3), we could get the change of state of ball

$$
\mathrm{X}(\mathrm{t})=\phi X(t-1)
$$

In this equation, $\phi=\left[\begin{array}{ll}1 & T \\ 0 & 1\end{array}\right]$ 。

Kalman filter using feedback control to estimate the process state: filter estimation state at a time, then measure variables with noises to obtain feedback. Therefore, the Kalman filter could be divided into two parts: estimated phase and status update phase[4].

For $\mathrm{X}(\mathrm{t})$ 's covariance $\mathrm{P}(\mathrm{t})$ :

$$
\mathrm{P}(\mathrm{t})=\phi \mathrm{P}(\mathrm{t}-1) \phi^{\prime}+Q
$$

$\mathrm{Q}$ is the covariance of the system, $\phi$ 'is transport matrix of $\phi$.

For the observation results of digital camera:

$$
Y(t)=H \cdot X(t)+u(t)
$$

$\mathrm{u}(\mathrm{t})$ is interference of digital camera. Because it comes from the precision of the camera, so it is the white Gaussian noise. $\mathrm{H}=\left[\begin{array}{lll}1 & 0 & 0\end{array}\right]$.

Let $X^{*}(t)$ be optimal value of ball's status with observation,

$X^{*}(t)=\mathrm{X}(\mathrm{t})+K_{g}(t) \cdot(Y(t)-\phi X(t))$

$K_{g}$ is Kalman gain

$$
K_{g}=P(t) H^{\prime} \cdot\left(H P(t) H^{*}+R\right)^{-1}
$$

For $X^{*}(t)^{\text {ss covariance }}$

$$
P^{*}(t)=\left(I-K_{g}(k) H\right) \cdot P(k)
$$

I is a matrix full of 1 
Thus we have $X^{*}(t)$ which could use in the strategy, and we also get $P^{*}(t)$ to instead of $\mathrm{P}(\mathrm{t}-1)$ to compute the status at time $t+1$. Because the program only saves $\mathrm{P}(\mathrm{t}-1)$ in the internal storage, so the operating efficiency of the program is very high.

The flow chart of the whole program is as the fig 1 .

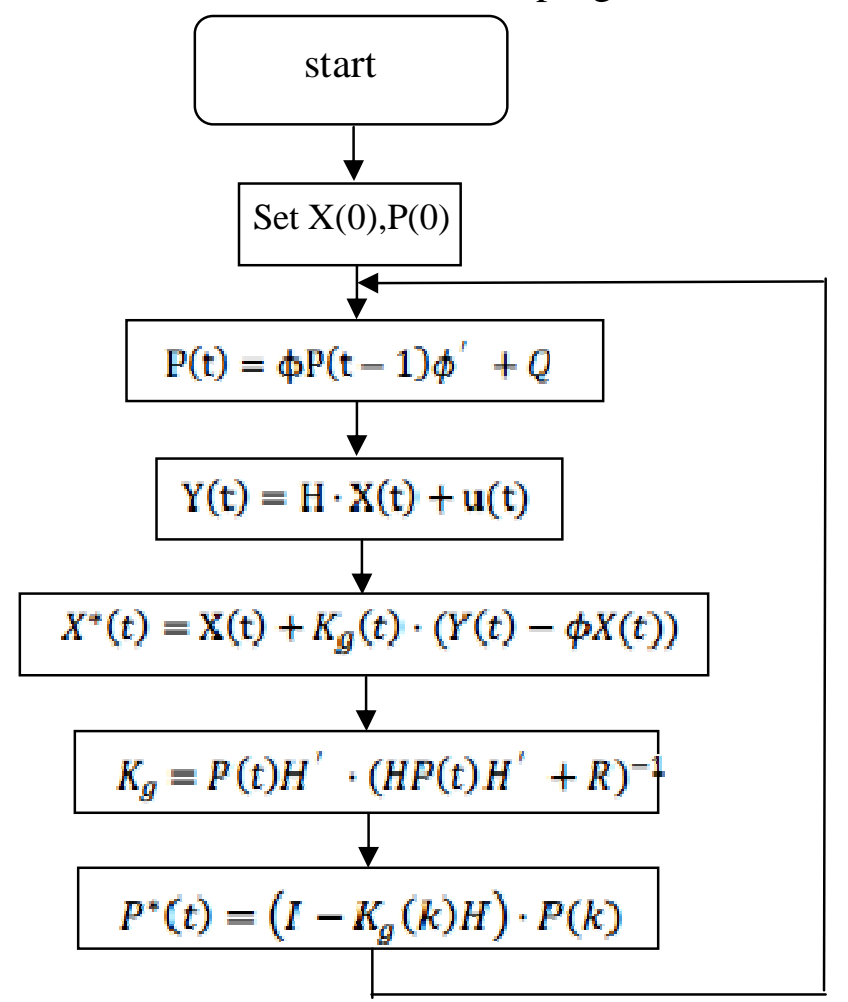

Fig.1 flows chart of the whole program

\section{Using Kalman filter to implement prediction of ball's position}

This system using $\mathrm{C}++$ to implement in the RobotSoccer v1.5a platform. In the real competition environment, $\mathrm{X}(\mathrm{t})=\left[\begin{array}{cc}50 & 47 \\ 0 & 0\end{array}\right]$ at time 0.50 and 47 is the initial coordinate of the ball in the system. It is totally trust in the data when $\mathrm{P}(0)=0$, which is not conducive to subsequent calculation. So we set $\mathrm{P}(0)$ as 10 at the start of system. In order to introduce the effectiveness and superiority, we present you two experiment.

Experiment 1, set a specific environment, only test the result of the predicted function of the location of the ball. The game field is as the fig 2. On the top right of the field, a yellow robot stops after giving the ball an initial velocity in the direction of underneath. The robot in the kick-off circle will chase the ball and other 8 robots would stand still. The fig 3 shows the test result without optimizing by the function of the Kalman filter. As we can tell, when the robots are getting close to the ball in the direction of $\mathrm{X}$ axis, it has already overpassed the location at the direction of $\mathrm{Y}$ axis. It is because of the previous inaccuracy of collecting data for the location of the ball by the acquisition function, it is also caused by the scale inaccuracy of the predicted function of the location next moment, and the slowness of updating. That is the point what the function of Kalman filter have already optimized and the result of the optimization is as the fig 4. Obviously we can see that after optimizing by the function of Kalman filter, when robots are to get the ball, they are basically at the same location in the direction of Y-axis, and the robots have already turned to the ball and adjusted their position to attack. 


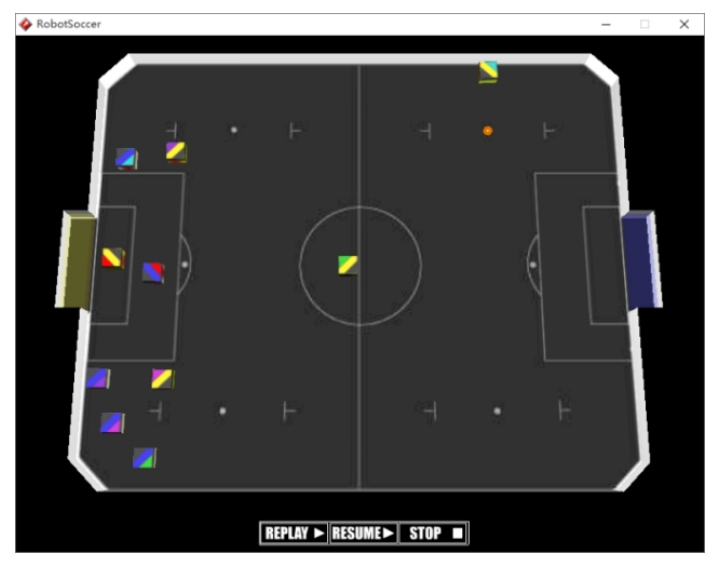

Fig.2 Initial state of the experiment

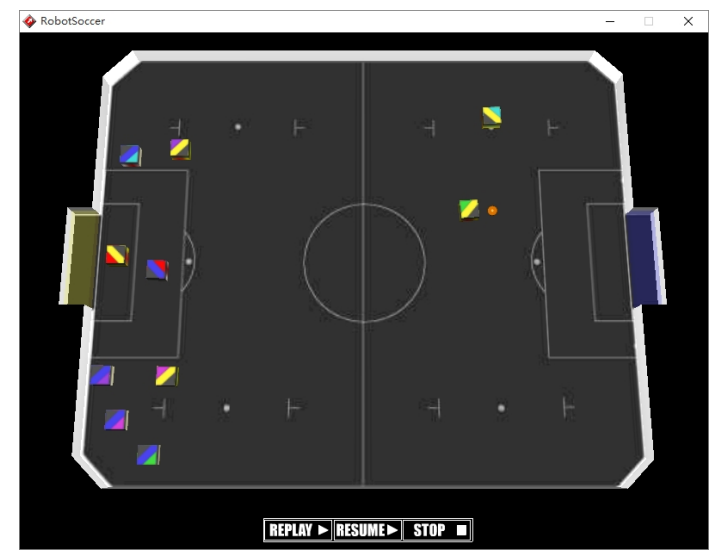

Fig.3 Before optimize by Kalman filter algorithm

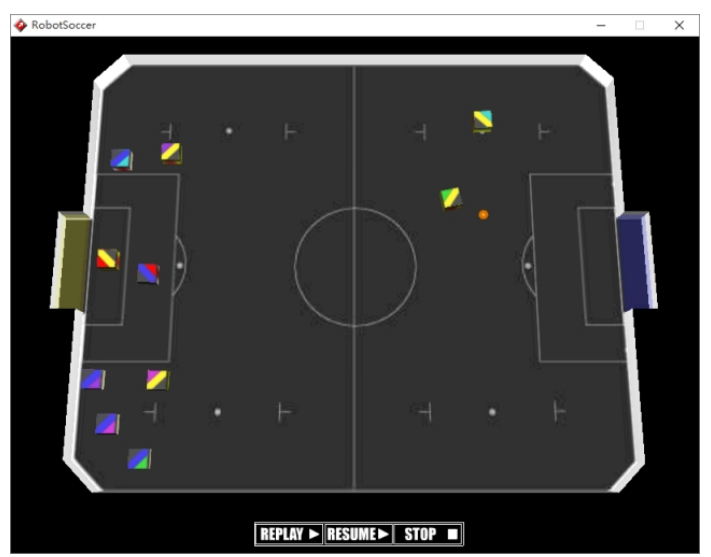

Fig.4 After optimize by Kalman filter algorithm

When blue team hold the offence chance at the baseline of the yellow team's half field, blue team will send a member outside of the penalty area. After failing to attack the goal, in most conditions the ball will bounce to the position of penalty mark. This moment, the "ambuscade" will go forward and "shot". As the fig 5 shows, forming a great chance to goal. This tactics is frequently used and efficient. However, it requires robots with high and accurate judgement and go forward and shoot at the right time. In this condition, the function of Kalman fliter has great effect. By using the way of Kalman Fliter proposed in the text, robots can locate the ball accurately, and make the robot blow can shoot more directly at the right time, so that increase the rate of goal.

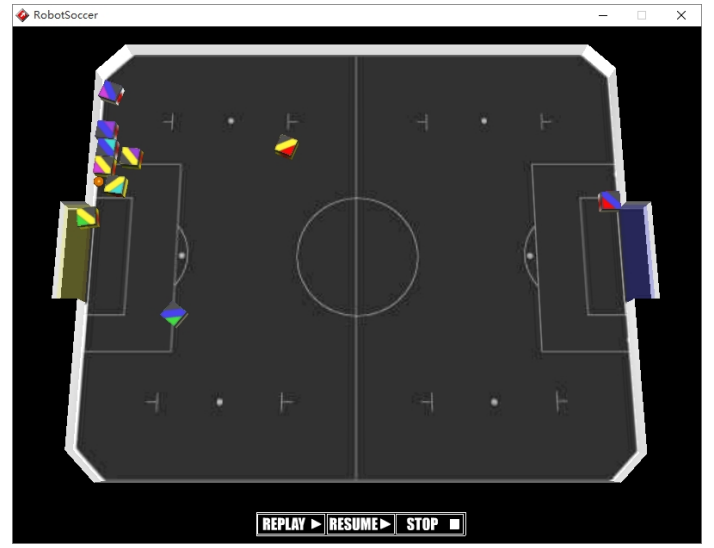

Fig.5 a common offensive strategy

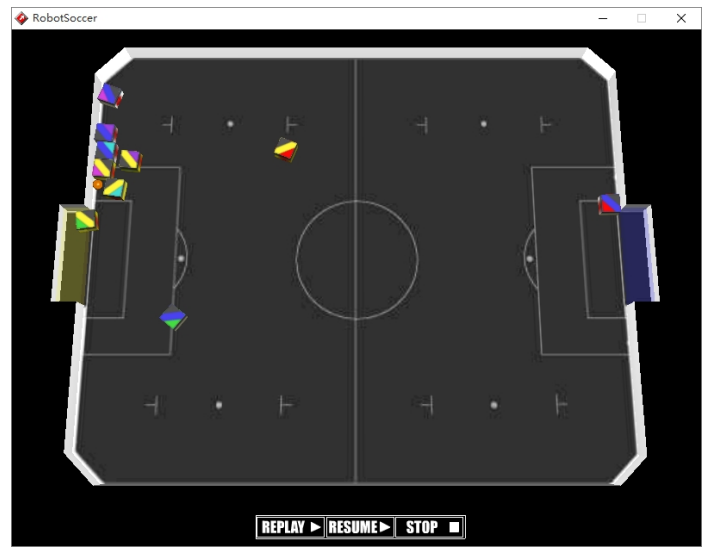

Fig.6 a great scoring opportunity 


\section{Summary}

Kalman filter is a widely-used function, and it plays significant roles in air, space and radar fields. This paper illustrates the application of Kalman filter on the robot soccer competition by using Kalman filter to eliminate the noise in the data from system, and doing dynamic and actual regression analysis, in order to make the robots more accurately determine the position of ball. This system won the second prize in the simulation group of FIRA in China robot competition \& RoboCup Open. It validated the feasibility and effectiveness of the Kalman filter application on robots soccer games. We hope we can observe the opponents' robots position in the future research, thus we can control the ball, and even the entire game by speculating the opponents' robots.

\section{Acknowledgements}

This paper is jointly sponsored by the Education and Teaching Project for undergraduate scientific research plan of Education Committee of the Beijing Municipality. (PXM2015_014224_000050), by Opening Project of Beijing Key Laboratory of Internet Culture and Digital Dissemination Research (ICDD201507).

\section{References}

[1] Information on http://fira.net

[2] GAO Dazhi, ZHANG Chunhui, XU Xinhe, Robot soccer-The new field of intelligent robot[J], in: Robot 1998, 20(4):309-314

[3] Asada M , Kitano H, NodaI, etal. RoboCup: today and tomorrow- what we have learned [J]. Artificial Intelligence, 1999, 110 (2):193 - 214.

[4] DONG Xiaojie, CHENG Qijun, Application of Kalman Filter in Soccer Robot Self-lization Equipment Manufacturing Technology, 2008,(2):45-48 